IJMMS 31:7 (2002) 387-406

PII. S0161171202013248

http://ijmms.hindawi.com

(c) Hindawi Publishing Corp.

\title{
NEW VERSIONS OF THE NYMAN-BEURLING CRITERION FOR THE RIEMANN HYPOTHESIS
}

\author{
LUIS BÁEZ-DUARTE
}

Received 15 May 2001 and in revised form 19 February 2002

Let $\rho(x)=x-[x], \chi=\chi_{(0,1)}, \lambda(x)=\chi(x) \log x$, and $M(x)=\sum_{k \leq x} \mu(k)$, where $\mu$ is the Möbius function. Norms are in $L_{p}(0, \infty), 1<p<\infty$. For $M_{1}(\theta)=M(1 / \theta)$ it is noted that $\zeta(s) \neq 0$ in $R s>1 / p$ is equivalent to $\left\|M_{1}\right\|_{r}<\infty$ for all $r \in(1, p)$. The space $\mathscr{B}$ is the linear space generated by the functions $x \mapsto \rho(\theta / x)$ with $\theta \in(0,1]$. Define $G_{n}(x)=$ $\int_{1 / n}^{1} M_{1}(\theta) \rho(\theta / x) \theta^{-1} d \theta$. For all $p \in(1, \infty)$ we prove the two arithmetical versions of the Nyman-Beurling theorem: (I) $\left\|M_{1}\right\|_{p}<\infty$ implies $\lambda \in \overline{\mathscr{S}}^{L p}$, and $\lambda \in \overline{\mathscr{B}}^{L p}$ implies $\left\|M_{1}\right\|_{r}<\infty$ for all $r \in(1, p)$. (II) $\left\|G_{n}-\lambda\right\|_{p} \rightarrow 0$ implies $\zeta(s) \neq 0$ in $\Re s \geq 1 / p$, and $\zeta(s) \neq 0$ in $\Re s>1 / p$ implies $\left\|G_{n}-\lambda\right\|_{r} \rightarrow 0$ for all $r \in(1, p)$.

2000 Mathematics Subject Classification: 11Mxx.

\section{Introduction}

1.1. Preliminaries and notation. For every $p \in[1, \infty]$ we canonically imbed $L_{p}(0,1)$ in $L_{p}(0, \infty)$. The conjugate index is always denoted by $q:=p /(p-1)$. Let $\rho(x):=$ $x-[x]$ stand throughout for the fractional part of the real number $x$, and $\chi:=\chi(0,1]$ is the characteristic function of the set $(0,1]$. We define the function $\lambda$ by

$$
\lambda(x):=\chi(x) \log x
$$

For every $a>0$ the operator $K_{a}$ given by

$$
K_{a} f(x):=f(a x),
$$

acts continuously on every $L_{p}(0, \infty)$ to itself, for $1 \leq p<\infty$.

$\mathscr{A}$ is the vector space of functions $f$ of the form

$$
f(x)=\sum_{k=1}^{n} c_{k} \rho\left(\frac{\theta_{k}}{x}\right),
$$

with $n \in \mathbb{N}, c_{k} \in \mathbb{C}, \theta_{k}>0,1 \leq k \leq n$. For $E \subseteq(0, \infty)$, denote by $\mathscr{A}_{E}$ the subspace of $\mathscr{A}$ where the $\theta_{k} \in E$. In particular we let $\mathscr{B}=\mathscr{A}_{(0,1]}$. $\mathscr{C}$ is the subspace of $\mathscr{B}$ resulting from requiring that

$$
\sum_{k=1}^{n} c_{k} \theta_{k}=0
$$

Clearly,

$$
\mathscr{C} \subset \mathscr{B} \subset A \subset L_{p}(0, \infty) \text { for } 1<p \leq \infty .
$$


Note that functions in $\mathscr{C}$ vanish in $(1, \infty)$, so $\mathscr{C} \subset L_{p}(0,1)$ for $1 \leq p \leq \infty$. $\mathscr{A}$ is invariant under any $K_{a}, a>0$, while $\mathscr{B}$ and $\mathscr{C}$ are invariant under $K_{a}$ for $a \geq 1$.

Recall the usual arithmetical functions $M$ and $g$ given by

$$
\begin{aligned}
& M(x)=\sum_{k \leq x} \mu(k), \\
& g(x):=\sum_{k \leq x} \frac{\mu(k)}{k},
\end{aligned}
$$

where $\mu$ is the Möbius function. We will denote

$$
M_{1}(\theta):=M\left(\frac{1}{\theta}\right)
$$

It is classical number theory that both $M(x) x^{-1} \rightarrow 0$ and $g(x) \rightarrow 0$ as $x \rightarrow \infty$ are elementarily equivalent to the prime number theorem. A stronger, but still elementary, estimate is $M(x) \ll x(\log x)^{-2}$. (Heretofore elementary is to be understood in the traditional number theoretical sense, as no analytic function theory, no Fourier analysis.)

We also define the less common $\gamma$ and $H_{p}$ by

$$
\begin{aligned}
\gamma(x) & :=\sum_{k \leq x-1} \frac{M(k)}{k(k+1)}, \\
H_{p}(x) & =\int_{1}^{x} M(t) t^{-2 / p} d t .
\end{aligned}
$$

Summing (1.7) by parts we get

$$
g(n)=\frac{M(n)}{n}+\gamma(n), \quad(n \in \mathbb{N}),
$$

and trivially from $|M(x)| \leq x$

$$
g(x)=\frac{M(x)}{x}+\gamma(x)+O\left(\frac{1}{x}\right), \quad(x \in \mathbb{R}) .
$$

1.2. The weak Nyman-Beurling theorem. An easy consequence of Wiener's $L_{2}$ Tauberian theorem (cf. [19]) is that $\mathscr{A}$ is dense in $L_{2}(0, \infty)$ (see [3]). Nyman [17] for $L_{2}$ and Beurling [9] for general $L_{p}$ obtained the much deeper result.

THEOREM 1.1 (Nyman-Beurling). The Riemann zeta-function is free from zeroes in the half-plane $\sigma>1 / p, 1<p<\infty$, if and only if $\mathscr{C}$ is dense in the space $L_{p}(0,1)$, which is equivalent to $-\chi \in \overline{\mathscr{C}}^{L_{p}}$.

To prove this theorem, Beurling first noted that $\mathscr{C}$ is dense in $L_{p}$ if and only if $-\chi \in \overline{\mathscr{C}}^{L_{p}}$, then showed, quite simply, that $-\chi \in \overline{\mathscr{C}}^{L_{p}}$ implies $\zeta(s) \neq 0$ for $\mathfrak{R} s>1 / p$. The proof of the converse, which, in his own words, is less trivial, is by contradiction. If $-\chi \notin \overline{\mathscr{C}}^{L_{p}}$, then $\mathscr{C}$ is not dense in the space $L_{p}(0,1)$. But this, by a highly involved functional analysis argument, implies the existence of a zero with real part greater than $1 / p$. Later proofs of this fact are illuminating, but just as difficult (see $[7,8,11])$. 
The degree to which the apparent depth of the two sides of the proof is so starkly contrasting has led some authors to doubt the usefulness of the Nyman-Beurling approach (see, e.g., [15]), yet, it has led others to attempt to level off the two sides of the proof.

We say that $\phi$ is a generator if $\phi \in L_{p}(0, \infty)$ for all $p \in(1, \infty)$ and

$$
L_{p}(0,1) \subseteq \operatorname{span}_{L_{p}}\left\{K_{a} \phi\right\}_{a \geq 1}, \quad(1<p<\infty) .
$$

These generators are called strong generators in [3], and the term generator is applied when $a$ is allowed to range in $(0, \infty)$ in $(1.13) .-\chi$ is the simplest example of a generator (the minus sign is immaterial, but more convenient). The function $\lambda$ defined in (1.1) is also a generator since

$$
\frac{1}{a-1}\left(K_{a}-I\right) \lambda \stackrel{L_{p}}{\longrightarrow} x, \quad(a \downarrow 1),(1 \leq p<\infty) .
$$

Clearly, any generator $\phi$ may well take the place of $-\chi$ in Theorem 1.1. These considerations, together with the fact that

$$
f(x)=\frac{1}{x} \sum_{k=1}^{n} c_{k} \theta_{k}, \quad(x>1),
$$

for every $f \in \mathscr{B}$ as in (1.3), allow the following minor extension of the Nyman-Beurling theorem (Theorem 1.1), where reference to density of $\mathscr{C}$ or $\mathscr{B}$ is dropped.

THEOREM 1.2. Let $\phi$ be a generator and $p \in(1, \infty)$. Then $\zeta(s) \neq 0$ for $\mathfrak{R} s>1 / p$ if and only if $\phi \in \overline{\mathscr{B}}^{L p}$.

Obviously the above theorem implies the following weaker version.

THEOREM 1.3 (weak Nyman-Beurling theorem). Let $p \in(1, \infty)$ and $\phi$ be a generator. Then $\zeta(s) \neq 0$ for $\mathfrak{R} s>1 / p$ if and only if $\phi \in \overline{\mathscr{P}}^{L r}$ for all $r \in(1, p)$.

Direct proofs of this theorem for $\phi=-\chi$, not depending on deep functional analysis results, were achieved independently by Lee [14], and Balazard and Saias [6]. These proofs only make use of standard number theoretical techniques. Thus Lee, perhaps not altogether inappropriately, calls the weak Nyman-Beurling theorem an arithmetical version of Beurling's theorem. The only if part of these proofs depends on identifying natural approximations $f_{n}$, which we define as sequences in $\mathscr{C}$ or $\mathscr{B}$, such that this weak implication holds for all $p \in(1, \infty)$ :

$$
\left(\zeta(s) \neq 0,\left(\mathfrak{R} s>\frac{1}{p}\right)\right) \Longrightarrow\left(\left\|f_{n}-\phi\right\|_{r} \longrightarrow 0, \forall r \in(1, p)\right) .
$$

Balazard and Saias [6] asked the following natural question.

QUESTION 1.4. For a given specific natural approximation $\left\{f_{n}\right\}$, is it true for some or all $p \in(1, \infty)$ that the weak implication (1.16) can be substituted for the strong implication

$$
(\zeta(s) \neq 0,(\Re s>1 / p)) \Longrightarrow\left(\left\|f_{n}-\phi\right\|_{p} \longrightarrow 0\right) ?
$$


We will answer this question, mostly in the negative, in Section 4. The first such natural approximation $\left\{B_{n}\right\} \subset \mathscr{B}$ appeared in [2] defined by

$$
B_{n}(x):=\sum_{k=1}^{n} \mu(k) \rho\left(\frac{1}{k x}\right)-n g(n) \rho\left(\frac{1}{n x}\right) .
$$

This sequence arises rather naturally in more than one way: it is the unique answer to the problem of finding $f \in \mathscr{C}$ as in (1.3) with $\theta_{k}=1 / k$, and $f(k / n)=-1$ for $1 \leq k \leq$ $n-1$. Or it can also be seen as a truncation of the fundamental identity

$$
-1=\sum_{k=1}^{\infty} \mu(k) \rho\left(\frac{1}{k x}\right), \quad(x>0) .
$$

It is easily seen that $B_{n}(x)=-1$ in $[1 / n, 1]$, and using the prime number theorem [2, Theorem 2.1] we proved that

$$
\left\|\chi+B_{n}\right\|_{1} \rightarrow 0
$$

which led us to ask whether the strong or the weak implications, (1.16), and (1.17), were true for $f_{n}=B_{n}, 1<p \leq 2$. A mild positive answer was [2, Proposition 2.4] that $\zeta(s)$ has a nontrivial zero-free half-plane if and only if $\left\|\chi+B_{n}\right\|_{p} \rightarrow 0$ for some $p>1$, which conferred some legitimacy to the question. In related work Vasyunin [21], referring to earlier results of Nikolski [16], took up the study of the $L_{2}$ case in quite some depth for a $B_{n}$-related sequence $\left\{V_{n}\right\} \subset \mathscr{C}$ defined by

$$
V_{n}(x):=\sum_{k=1}^{n} \mu(k) \rho\left(\frac{1}{k x}\right)-g(n) \rho\left(\frac{1}{x}\right) .
$$

Vasyunin also conducted numerical studies leading him to state (sic) we can hardly hope that the series converges in the $L_{2}$-norm. That this is indeed the case was first proved in [3]. The sequence $\left\{S_{n}\right\} \subset \mathscr{B}$ defined by

$$
S_{n}(x):=\sum_{k=1}^{n} \mu(k) \rho\left(\frac{1}{k x}\right),
$$

perhaps the most natural in view of (1.19), is obviously $L_{2}$-equivalent to $\left\{V_{n}\right\}$ since $g(n) \rightarrow 0$. The relationship with $B_{n}$ is more complicated, however, since by Corollary 2.5 below the $L_{p}$-norm of $n g(n) \rho(1 / n x)$ is of order $|g(n)| n^{1 / q}$ which does not tend to zero if $\zeta(s)$ has a zero with real part $1 / p$, such being the case, of course if $p=2$. Furthermore $B_{n}$ is not a series, as defined in (4.24), while $V_{n}$ is the most natural series.

Lee [14] proved Theorem 1.3 using $V_{n}, 1<p \leq 2$, and, independently, Balazard and Saias [6] did likewise for $B_{n}$ and $S_{n}, 1<p<\infty$.

A further approximating sequence $\left\{F_{n}\right\} \subset \mathscr{C}$ promoted in [2] as the dual approximation, given by

$$
F_{n}(x):=\sum_{k=1}^{n}\left(M\left(\frac{n}{k}\right)-M\left(\frac{n}{k+1}\right)\right) \rho\left(\frac{k}{n x}\right)-\rho\left(\frac{1}{n x}\right),
$$

is of a different nature, as the $\theta_{k}$ are uniformly distributed in $(0,1)$ as $n \rightarrow \infty$. It is proved in [2] that $\left\|F_{n}+\chi\right\|_{1} \rightarrow 0$, and it can also be shown that $F_{n}(x) \rightarrow-1$ for $0<x \leq 1$. The following question is however open. 
QUESTION 1.5. Is $F_{n}$ a natural approximation?

1.3. Description of main results. It is classical knowledge that the Riemann hypothesis, an analytical statement about the location of the zeroes of the zeta function, is equivalent to purely arithmetical statements relative to the distribution of the prime numbers. A classical example is Littlewood's criterion: the Riemann hypothesis is equivalent to $M(x)=O\left(x^{1 / 2+\epsilon}\right)$ for all $\epsilon>0$. This criterion generalized to the half-planes $\Re s>1 / p$ leads to the following equivalence proved in Section 2 .

Proposition 1.6. For any $p \in(1, \infty)$ the following are equivalent:

(i) $\zeta(s) \neq 0$ for $\mathfrak{R} s>1 / p$;

(ii) $\left\|M_{1}\right\|_{p}<\infty$ for all $r \in(1, p)$.

It is this condition (ii) on the Möbius function, which in effect, if not always in name, takes the place of the Riemann hypothesis in this paper. This together with their simple and quasi-elementary proofs given in Section 3 has led us to characterize the following two theorems as arithmetical versions of the Nyman-Beurling theorem, in contrast with which, furthermore, the proofs for both the if and the only if implications are of nearly equivalent difficulty. These two theorems stand somewhere between the strong Theorem 1.2 and the weak Theorem 1.3.

THEOREM 1.7 (arithmetical Nyman-Beurling's theorem I). The following statements are true for all $p \in(1, \infty)$ :

(a) $\left\|M_{1}\right\|_{p}<\infty$ implies $\lambda \in \overline{\mathscr{B}}^{L_{p}}$;

(b) $\lambda \in \overline{\mathscr{B}}^{L_{p}}$ implies $\left\|M_{1}\right\|_{r}<\infty$ for all $r \in(1, p)$.

Next we introduce a new "natural approximation" $G_{n}$ which will be seen to belong in every ${\overline{A_{(1 / n, 1]}}}^{L p}, p \in(1, \infty)$, defined by

$$
G_{n}(x):=\int_{1 / n}^{\infty} M_{1}(\theta) \rho\left(\frac{\theta}{x}\right) \frac{d \theta}{\theta} .
$$

$G_{n}$ arises very naturally from an integrated form of the basic identity for the Möbius numbers which we will prove in Section 3, namely,

$$
\lambda(x)=\int_{0}^{1} M_{1}(\theta) \rho\left(\frac{\theta}{x}\right) \frac{d \theta}{\theta} .
$$

The above formula can be seen as a formal proof of the Riemann hypothesis since the integral shows the generator $\lambda$ as a limit of functions of $\mathscr{B}$. Then our second main result is the following theorem.

THEOREM 1.8 (arithmetical Nyman-Beurling theorem II). The following statements are true for all $p \in(1, \infty)$ :

(c) $\zeta(s) \neq 0, \mathfrak{R} s>1 / p$ implies $\left\|G_{n}-\lambda\right\|_{r} \rightarrow 0$ for all $r \in(1, p)$;

(d) $\left\|G_{n}-\lambda\right\|_{p} \rightarrow 0$ implies $\zeta(s) \neq 0, \mathfrak{R} s \geq 1 / p$.

Finally, in Section 4, we explore the delicate gap between the weak and strong forms of the Nyman-Beurling theorem. We will show that all the natural approximations $B_{n}, V_{n}, S_{n}, F_{n}$, and $G_{n}$ diverge in $L_{2}$. We also study the general $L_{p}$ case. The most 
interesting conclusion is this: if the Riemann hypothesis were not true, and $1 / p=$ $\sup \{\Re s \mid \zeta(s)=0\}$, then $S_{n}, V_{n}$, and $G_{n}$ would also diverge in $L_{p}$ provided there is a zero of real part $1 / p$. We have not decided the question for $B_{n}$ and $F_{n}$.

2. Technical lemma and preliminary propositions. Throughout this section $1<$ $p<\infty$. Some of the results herein may be part of the common folklore and/or stated in less general form than is possible. They are listed here however for the sake of completeness and readability. We thank A. M. Odlyzko for his generous help in these matters.

2.1. Technical lemma. It is assumed that $f$ is a locally bounded complex-valued function defined on $[1, \infty)$, whose Mellin transform $\tilde{f}$, defined here as

$$
\tilde{f}(s):=\int_{1}^{\infty} f(x) x^{-s-1} d x
$$

has a finite abscissa of convergence $\alpha=\alpha_{f}$.

LEMMA 2.1 (order lemma). If $\tilde{f}(s)$ has a pole at $s_{0}=\sigma_{0}+i t_{0}$ in a meromorphic extension to a possibly larger half-plane, then $f(x) \neq o\left(x^{\sigma_{0}}\right)$.

Proof. This is just an adaptation of the proof of $M(x) \neq o(\sqrt{x})$ in [20]. Assume by contradiction that $f(x)=o\left(x^{\sigma_{0}}\right)$. A fortiori $f(x)=O\left(x^{\sigma_{0}}\right)$, so that the integral in (2.1) would actually converge in $\mathfrak{R} s>\sigma_{0}$. Now let $s=\sigma+i t_{0}$ with $\sigma \downarrow \sigma_{0}$. If $m \geq 1$ is the order of the pole, then we have

$$
\tilde{f}\left(\sigma+i t_{0}\right) \sim \frac{C}{\left(\sigma-\sigma_{0}\right)^{m}}, \quad\left(\sigma \rightarrow \sigma_{0}\right),
$$

for some $C \neq 0$. On the other hand the little $o$ condition implies there is an $A>1$ such that $|f(x)|<(|C| / 2) x^{\sigma_{0}}$ for $x>A$, so that splitting the right-hand side integral in (2.1) as $\int_{1}^{A}+\int_{A}^{\infty}$ we obtain

$$
\left|\tilde{f}\left(\sigma+i t_{0}\right)\right| \leq O_{A}(1)+\frac{|C|}{2\left(\sigma-\sigma_{0}\right)},
$$

which contradicts (2.2).

LEMMA 2.2 (oscillation lemma). Let $f$ be real valued. If $\alpha=\alpha_{f}$ is not a singularity of $\tilde{f}(s)$ then for any $\epsilon>0$

$$
\begin{aligned}
\limsup _{x \rightarrow \infty} f(x) x^{-\alpha+\epsilon} & =+\infty, \\
\liminf _{x \rightarrow \infty} f(x) x^{-\alpha+\epsilon} & =-\infty .
\end{aligned}
$$

In particular, $f(x)$ changes sign an infinite number of times as $x \rightarrow \infty$.

Proof. It is obviously enough to deal with only one of the above relations. So assume that (2.5) is false. Then for some $\epsilon>0$ there is a $C$ such that

$$
C x^{\alpha-\epsilon}-f(x) \geq 0, \quad(x>1) .
$$


Therefore,

$$
\int_{1}^{\infty}\left(C x^{\alpha-\epsilon}-f(x)\right) x^{-s-1} d x=\frac{B}{s-\alpha+\epsilon}-\tilde{f}(s)
$$

is not singular at $s=\alpha$, but clearly $\alpha$ is also the abscissa of convergence of the lefthand side integral above, which contradicts the theorem that the Laplace transform of a positive measure has a singularity on the real axis at the abscissa of convergence [22, Theorem 5.b].

LEMMA 2.3. Let $F:[1, \infty) \rightarrow \mathbb{C}$ be locally integrable. If $\int_{1}^{x} F(t) d t \neq o\left(x^{1 / q}\right)$, then $\|F\|_{p}=\infty$.

Proof. It is obviously enough to consider that $F \geq 0$. By hypothesis there exists some $\epsilon>0$, and an unbounded set $E \subset[1, \infty)$ such that

$$
\int_{1}^{y} F(t) d t>\epsilon y^{1 / q}, \quad(\forall y \in E) .
$$

Now take an arbitrary $x>1$. It is easy to see that there exists $y \in E$ such that $y>x$ and

$$
2 \int_{1}^{x} F(t) d t<\epsilon y^{1 / q}<\int_{1}^{y} F(t) d t
$$

so that

$$
\int_{x}^{y} F(t) d t>\frac{\epsilon}{2} y^{1 / q}
$$

But Hölder's inequality gives

$$
\int_{x}^{y} F(t) d t \leq(y-x)^{1 / q}\left(\int_{x}^{y}(F(t))^{p} d t\right)^{1 / p},
$$

which, introduced in (2.10), yields

$$
\int_{x}^{y}(F(t))^{p} d t>\frac{\epsilon}{2}\left(\frac{y}{y-x}\right)^{1 / q}>\frac{\epsilon}{2} .
$$

\subsection{Some preliminary propositions}

Proposition 2.4. The following Mellin transforms are valid at least in the halfplanes indicated:

$$
\begin{gathered}
\int_{1}^{\infty} M(x) x^{-s-1} d x=\frac{1}{s \zeta(s)}, \quad(\Re s>1), \\
\int_{1}^{\infty}(x g(x)) x^{-s-1} d x=\frac{1}{(s-1) \zeta(s)}, \quad(\mathfrak{R} s>1), \\
\int_{1}^{\infty}(x \gamma(x)) x^{-s-1} d x=\frac{1}{s(s-1) \zeta(s)}+\omega(s), \quad(\Re s>1), \\
\int_{1}^{\infty} H_{p}(x) x^{-s-1} d x=\frac{1}{s(s+2 / p-1) \zeta(s+2 / p-1)}, \quad\left(\mathfrak{R} s>\frac{2}{q}\right),
\end{gathered}
$$

where $\omega(s)$ is analytic in $\Re s>0$. 
Proof. As in Titchmarsh's monograph [20] we write for $\mathfrak{R} s>1$

$$
\begin{aligned}
\frac{1}{\zeta(s)} & =\sum_{n=1}^{\infty}(M(n)-M(n-1)) n^{-s} \\
& =\sum_{n=1}^{\infty} M(n)\left(n^{-s}-(n+1)^{-s}\right) \\
& =s \sum_{n=1}^{\infty} M(n) \int_{n}^{n+1} x^{-s-1} d x \\
& =s \int_{1}^{\infty} M(x) x^{-s-1} d x .
\end{aligned}
$$

This proves (2.13). Proceed likewise with

$$
\frac{1}{\zeta(s)}=\sum_{n=1}^{\infty}(g(n)-g(n-1)) n^{-s+1}, \quad(\Re s>1),
$$

to obtain (2.14). Now using relation (1.12) between $M(x), g(x)$, and $\gamma(x)$ subtract the preceding two Mellin transforms to get (2.15). Finally, from definition (1.10) and the trivial $|M(x)| \leq x$ we deduce $H_{p}(x) \ll x^{2 / q}$. Next note that $H_{p}$ is continuous and piecewise differentiable, which justifies the following integration by parts at least for Rs $>2 / q$ :

$$
\int_{1}^{\infty} H_{p}(x) x^{-s-1} d x=\frac{1}{x} \int_{1}^{\infty} M(x) x^{-s-2 / p} d x
$$

Now apply (2.13) to arrive at (2.16).

The following corollary is an immediate consequence of the above Mellin transforms, and the order and oscillation lemmas (Lemmas 2.1 and 2.2).

COROLLARY 2.5. Each one of the functions $M(x), g(x), \gamma(x), H_{p}(x)$, change sign infinitely often as $x \rightarrow \infty$. Furthermore, if $\zeta(s)$ has some zero on the line $\mathfrak{R} s=1 / p$, then $M(x) \neq o\left(x^{1 / p}\right), g(x) \neq o\left(x^{-1 / q}\right), \gamma(x) \neq o\left(x^{-1 / q}\right)$, and $H_{p}(x) \neq o\left(x^{1 / q}\right)$.

REMARK 2.6. In particular, $M(x) \neq o(\sqrt{x})$ (see [20]). Sharper results are of course known, for example, that the Mertens hypothesis is false, with $M(x)$ oscillating beyond $\pm \sqrt{x}$. This was proven by Odlyzko and te Riele [18].

Some further properties of $\gamma(x)$ needed later are gathered here.

LEMMA 2.7. The function $\gamma$ satisfies:

(i) $\gamma(n) \rightarrow 0$ as $n \rightarrow \infty$,

(ii) $\gamma(n)=\int_{1}^{n} M(t) t^{-2} d t,(n \in \mathbb{N})$,

(iii) $\int_{1}^{\infty} M_{1}(\theta) d \theta=\int_{1}^{\infty} M(t) t^{-2} d t=0$. This integral converges absolutely.

REMARK 2.8. In [4] we showed that the existence of $\lim _{n \rightarrow \infty} \gamma(n)$ is elementarily equivalent to the prime number theorem.

Proof of Lemma 2.7. The prime number theorem and (1.11) yield (i). Decomposing the integral in (ii) in the intervals $(k, k+1)$ one gets (ii). Letting $n \rightarrow \infty$ in (ii) yields (iii). The absolute convergence follows from the elementary estimate $M(x) \ll$ $x(\log x)^{-2}$. 
The result on $H_{p}(x)$ in Corollary 2.5 begets some important consequences for the norms of $M_{1}$.

Proposition 2.9. If $\zeta(s)$ has a zero on the line $\mathfrak{R} s=1 / p$, then

$$
\left\|M_{1}\right\|_{p}=\infty .
$$

REMARK 2.10. Note therefore that

$$
\left\|M_{1}\right\|_{p}<\infty \text { implies }(\zeta(s) \neq 0 \text { for } \mathfrak{R} s \geq 1 / p) .
$$

Proof of Proposition 2.9. Take $F(x):=M(x) x^{-2 / p}$. Then $F(x) \neq o\left(x^{1 / q}\right)$ by Corollary 2.5 , so the divergent integral lemma (Lemma 2.3 ) yields

$$
\left\|M_{1}\right\|_{p}^{p}=\int_{1}^{\infty}|M(x)|^{p} x^{-2} d x=\infty .
$$

REMARK 2.11. Since $\zeta(s)$ has roots in the critical line the above corollary tells us that

$$
\left\|M_{1}\right\|_{2}=\infty \text {. }
$$

Using far more refined techniques Konyagin and Popov [13] have shown a stronger result in the case $p=2$, namely

$$
\int_{1}^{x}|M(t)|^{2} t^{-2} d t \gg \log x .
$$

Now we can prove Proposition 1.6 to the effect that $\zeta(s) \neq 0$ in $\Re s>1 / p$ if and only if $\left\|M_{1}\right\|_{p}<\infty$.

Proof of Proposition 1.6. An extension of Littlewood's well-known criterion for the Riemann hypothesis is that condition (i) is equivalent to

$$
M(x) \ll x^{1 / p^{\prime}} \quad \forall p^{\prime} \in(1, p),
$$

(see [10, Proposition IV.21]), so choose $p^{\prime}$ with $r<p^{\prime}<p$, and then it is obvious how (i) implies (ii). Now we prove that not (i) implies not (ii). So assume there is an $s_{0}$ with $\mathfrak{R} s_{0}=1 / p_{1}>1 / p$ and $\zeta\left(s_{0}\right)=0$. Then by Proposition 2.9

$$
\left\|M_{1}\right\|_{p_{1}}=\infty,
$$

but (probability space) $\left\|M_{1}\right\|_{p_{1}} \leq\left\|M_{1}\right\|_{r}$ since $p_{1}<r<p$.

Define the Riemann abscissa $\beta$ by

$$
\beta:=\sup _{\zeta(s)=0} R s
$$

Nothing is known beyond $1 / 2 \leq \beta \leq 1$. We do know however that, on the one hand there are no zeroes on the line $\mathfrak{R} s=1$ and $\left\|M_{1}\right\|_{1}<\infty$, since $M(t) \ll t(\log t)^{-2}$, and, on the other hand there are zeroes on the line $\mathfrak{R} s=1 / 2$ and $\left\|M_{1}\right\|_{2}=\infty$. One could rightly ask the following question. 
QUESTION 2.12. For $\beta \in(1 / 2,1)$, is it true that $\left\|M_{1}\right\|_{1 / \beta}<\infty$ if and only if $\zeta(s) \neq 0$ for $R s=\beta$ ?

3. Arithmetical versions of the Nyman-Beurling theorem. We define an operator $T$ acting on all $L_{p}(0, \infty), p \in(1, \infty)$, by

$$
T f(x):=\int_{0}^{\infty} f(\theta) \rho\left(\frac{\theta}{x}\right) \frac{d \theta}{\theta},
$$

noting that the above integral converges absolutely for $f \in L_{p}(0, \infty)$ by Hölder's inequality. Now we show that $T$ is of type $(p, p)$. This does not follow, as could be expected, from the convolution form of the operator, on account of the difference between the measures $d x$ and $d x / x$ in $(0, \infty)$.

LEMMA 3.1. For every $p \in(1, \infty)$ the operator $T$ is a continuous operator from $L_{p}(0, \infty)$ to itself.

Proof. Let $f \geq 0$ and $x>0$, then splitting the range of integration at $x$ in (3.1) we get

$$
T f(x) \leq \frac{1}{x} \int_{0}^{x} f(\theta) d \theta+\int_{x}^{\infty} f(\theta) \frac{d \theta}{\theta} .
$$

The result now emerges from the well-known, elementary Hardy inequalities (see [12, Theorems 327, 328]).

The next result establishes the relevance of $T$ for the Nyman-Beurling approach.

Proposition 3.2. For any $p \in(1, \infty)$, and an interval $E \subseteq(0, \infty)$ the range of $T$ satisfies

$$
{\overline{T L_{p}(E)}}^{L_{p}}={\overline{\mathscr{A}_{E}}}^{L_{p}} .
$$

REMARK 3.3. For every $f \in L_{p}(0, \infty), T f$ is continuous, so the closure operation on the left-hand side above is necessary. However, for the purpose immediately at hand of proving Theorem 1.7 we only need

$$
T L_{p}((0,1]) \subset \overline{\mathscr{B}}^{L p} .
$$

REMARK 3.4. If $f \in L_{p}(0,1)$ for some $p \in(1, \infty)$ and

$$
\int_{0}^{1} f(\theta) d \theta=0
$$

then $T f \in \overline{\mathscr{C}}^{L p}$. This is the case for $f=M_{1}$ by Lemma 2.7(iii).

Proof of Proposition 3.2. Fix $p \in(1, \infty)$. For any bounded interval $[a, b] \subseteq E$

$$
T \chi_{[a, b]}(x)=\int_{a}^{b} \rho\left(\frac{\theta}{x}\right) \frac{d \theta}{\theta}
$$

is a proper Riemann integral for each $x>0$. Let $\theta_{n, k}:=a+(b-a)(k / n)$, and

$$
s_{n}(x):=\frac{b-a}{n} \sum_{k=1}^{n} \frac{1}{\theta_{n, k}} \rho\left(\frac{\theta_{n, k}}{x}\right) .
$$


The Riemann sums $s_{n}(x) \in \mathscr{A}$ and $s_{n}(x) \rightarrow T \chi_{[a, b]}(x)$ for each $x>0$. Furthermore, it is trivial to see that $s_{n}(x) \leq(b-a) / a$ for all $x>0$, whereas $s_{n}(x)=(b-a) / x$ when $x>b$, so that

$$
s_{n}(x) \leq \frac{b-a}{a} \chi_{(0, b]}(x)+\frac{b-a}{x} \chi_{(b, \infty)}(x) .
$$

Hence $\left\|s_{n}-T \chi_{[a, b]}\right\|_{p} \rightarrow 0$. By Proposition 3.2 we conclude

$$
T \chi_{[a, b]} \in \overline{\mathscr{A}}_{E}^{L p},
$$

which the time honored density argument and the continuity of $T$ convert into (3.4), and, a fortiori, $\overline{T L}_{p}(E) \subseteq \overline{\mathscr{A}}_{E}^{L_{p}}$. To finish the proof of (3.1) we need to show that each function $\rho(\alpha / x), \alpha \in E$ is in ${\overline{T L_{p}(E)}}^{L_{p}}$. This is achieved as follows: for $\alpha \neq a$ take $1>h \downarrow 0$. Clearly

$$
\frac{1}{\max (\alpha, x)} \geq \frac{1}{\alpha h} \int_{\alpha(1-h)}^{\alpha} \rho\left(\frac{\theta}{x}\right) \frac{d \theta}{\theta} \rightarrow \rho\left(\frac{\alpha}{x}\right), \quad(\text { a.e. } x) .
$$

By (3.9) $(\alpha h)^{-1} T \chi_{[\alpha(1-h), \alpha]} \in{\overline{A_{E}}}^{L_{p}}$, and the above inequalities show it converges in $L_{p^{-}}$ norm to the function $\rho(\alpha / x)$. If $\alpha=a$ the modification to the above proof is obvious.

We next introduce the essential, elementary identity.

LEMMA 3.5. For every $x>0$,

$$
\chi_{(1, \infty)}(x) \log x=\int_{1}^{x} M(t)\left[\frac{x}{t}\right] \frac{d t}{t} .
$$

Proof. Here we denote $\chi(S)=1$ if the statement $S$ is true, otherwise $\chi(S)=0$. We start from the well-known elementary identity

$$
\chi_{[1, \infty)}(t)=\sum_{n=1}^{\infty} M\left(\frac{t}{n}\right),
$$

which we multiply by $1 / t$ and integrate, thus

$$
\begin{aligned}
\chi_{[1, \infty)}(x) \log x & =\int_{0}^{x} \sum_{n=1}^{\infty} M\left(\frac{t}{n}\right) \frac{d t}{t} \\
& =\sum_{n=1}^{\infty} \int_{0}^{x} M\left(\frac{t}{n}\right) \frac{d t}{t} \\
& =\sum_{n=1}^{\infty} \int_{0}^{x / n} M(t) \frac{d t}{t} \\
& =\int_{0}^{x} M(t) \sum_{n=1}^{\infty} x\left(t \leq \frac{x}{n}\right) \frac{d t}{t} \\
& =\int_{0}^{x} M(t)\left[\frac{x}{t}\right] \frac{d t}{t} .
\end{aligned}
$$


Proposition 3.6. For every $x>0$, the following identity holds true as an absolutely convergent integral, without any assumptions on the $L_{p}$-norms of $M_{1}$ :

$$
\lambda(x)=\int_{0}^{1} M_{1}(\theta) \rho\left(\frac{\theta}{x}\right) \frac{d \theta}{\theta} .
$$

Proof. The upper limit of integration in (3.11) can trivially be substituted by $\infty$, so we get

$$
\begin{aligned}
\chi_{(1, \infty)}(x) \log x & =\int_{1}^{\infty} M(t)\left(\frac{x}{t}-\rho\left(\frac{x}{t}\right)\right) \frac{d t}{t} \\
& =-\int_{1}^{\infty} M(t) \rho\left(\frac{x}{t}\right) \frac{d t}{t}
\end{aligned}
$$

from Lemma 2.7(iii), and the (absolute) convergence of the last integral, again due to $M(t) \ll t(\log t)^{-2}$. Now make the change of variables $t=1 / \theta$, and in the formula obtained substitute $x \mapsto 1 / x$.

REMARK 3.7. In [4] we show that the existence of

$$
\lim _{\epsilon \rightarrow 0} \int_{\epsilon}^{1} M_{1}(\theta) \rho\left(\frac{\theta}{x}\right) \frac{d \theta}{\theta}
$$

is elementarily equivalent to the prime number theorem.

3.1. The proofs of the main theorems. We now prove our first main result, Theorem 1.7.

PRoof. We prove statement (a): if $\left\|M_{1}\right\|_{p}<\infty$, then $\lambda=T M_{1} \in T L_{p}(0,1) \subset{\overline{\mathscr{A}_{(0,1)}}}^{L_{p}}$ $=\overline{\mathscr{S}}^{L_{p}}$ by (3.14), Lemma 3.1, and (3.4). To prove (b), note that if $\lambda \in \overline{\mathscr{B}}^{L_{p}}$, then $\chi \in$ $\overline{\mathscr{B}}^{L p}$ as remarked in (1.14). Then by the easy sufficiency part of the Nyman-Beurling theorem (Theorem 1.2) $\zeta(s) \neq 0$ for $\mathfrak{R} s>1 / p$, and this implies by Proposition 1.6 that $\left\|M_{1}\right\|_{r}<\infty$ for all $r \in(1, p)$.

REMARK 3.8. The proof of (a) is elementary, and, interestingly, it corresponds to the hard necessity part of the Nyman-Beurling theorem (Theorem 1.2). Note however that the strong form of (a) is connected with the fact that the hypothesis implies by (2.21) that there are no zeroes of $\zeta(s)$ in $\Re s \geq 1 / p$. On the other hand (b), a weak statement, corresponding to the easy sufficiency part of the Nyman-Beurling theorem (Theorem 1.2), is proved essentially by the traditional argument.

We now prove our second main result, Theorem 1.8.

Proof. It is easy to see from Proposition 3.2 that $G_{n} \in{\overline{\mathbb{A}_{(1 / n, 1]}}}^{p}$ for all $p \in(1, \infty)$. Now let us prove statement (c). If $\zeta(s) \neq 0$ for $\mathfrak{R} s>1 / p$, then, by Proposition 1.6, $\left\|M_{1}\right\|_{r}<\infty$ for all $r \in(1, p)$. It is then clear that $G_{n}=T\left(M_{1} \chi_{(1 / n, 1]}\right) \stackrel{L_{r}}{\longrightarrow} \lambda$ by the $L_{p}$-continuity of $T$ (Lemma 3.1). To prove (d) we proceed by contradiction. Assume there is $s_{0}$ such that $\zeta\left(s_{0}\right)=0$ and $\Re s_{0}=1 / p_{1} \geq 1 / p$. Therefore $\gamma(n) \neq o\left(n^{-1 / q_{1}}\right)$ by 
Corollary 2.5. Now by definition (1.24) of $G_{n}$ and (ii) in Lemma 2.7 we have

$$
\begin{aligned}
\left\|G_{n}-\lambda\right\|_{p}^{p} & =\int_{0}^{\infty}\left|\int_{0}^{1 / n} M_{1}(\theta) \rho\left(\frac{\theta}{x}\right) \frac{d \theta}{\theta}\right|^{p} d x \\
& \geq \int_{1 / n}^{\infty}\left|\int_{0}^{1 / n} M_{1}(\theta) \rho\left(\frac{\theta}{x}\right) \frac{d \theta}{\theta}\right|^{p} d x \\
& =\int_{1 / n}^{\infty}\left|\frac{1}{x} \int_{0}^{1 / n} M_{1}(\theta) d \theta\right|^{p} d x \\
& =(p-1)^{-1}|\gamma(n)|^{p} n^{p-1},
\end{aligned}
$$

so that

$$
\left\|G_{n}-\lambda\right\|_{p} \geq(p-1)^{-1 / p}|\gamma(n)| n^{1 / q_{1}} \rightarrow 0 .
$$

REMARK 3.9. The proof of (c), a weak statement corresponding to the hard necessity part of the Nyman-Beurling theorem (Theorem 1.2), is easy and quasi elementary. On the other hand the proof of (d), a strong statement, corresponding to the easy sufficiency part of the Nyman-Beurling theorem, is rather easy, but not elementary.

REMARK 3.10. At least, formally we can apply the operators

$$
\mathscr{D}_{h}:=\frac{1}{h}\left(K_{(1+h)}-I\right)
$$

to (c), and let $h \downarrow 0$ to obtain the corresponding Balazard-Saias result for $S_{n}$ in [6]. The difficulty in formalizing this argument stems from the fact that, for $\mathscr{D}_{h}$ as an operator from $L_{p}$ to itself, $\left\|\mathscr{D}_{h}\right\| \rightarrow \infty$ except when $p=1$. A rigorous proof would be desirable.

3.2. Behavior of $G_{n}$ in $L_{1} . G_{n}$ also behaves nicely pointwise and in $L_{1}$, as the original natural approximations. To see this we first need a lemma.

LEMMA 3.11. For $\theta>n$,

$$
\int_{n}^{\infty} \rho\left(\frac{x}{\theta}\right) \frac{d x}{x^{2}} \ll \frac{\log \theta}{\theta}
$$

Proof of The Lemma. For $\theta>n$, we have

$$
\begin{aligned}
\int_{n}^{\infty} \rho\left(\frac{x}{\theta}\right) \frac{d x}{x^{2}} & =\frac{1}{\theta} \int_{n}^{\theta} \frac{d x}{x}+\int_{\theta}^{\infty} \rho\left(\frac{x}{\theta}\right) \frac{d x}{x^{2}} \\
& \leq \frac{\log \theta}{\theta}+\frac{1}{\theta} .
\end{aligned}
$$

Proposition 3.12. $G_{n}$ satisfies the properties

$$
\begin{gathered}
G_{n}(x)-\lambda(x) \longrightarrow 0, \quad(\forall x>0), \\
\int_{0}^{1}\left|G_{n}(x)-\lambda(x)\right| d x \longrightarrow 0 .
\end{gathered}
$$


Proof. The first statement follows easily from the fact that the integral in (3.14) is absolutely convergent. Changing variables in the first iterated integral below, we get

$$
\left\|G_{n}-\lambda\right\|_{1}=\int_{0}^{1}\left|\int_{0}^{1 / n} M_{1}(\theta) \rho\left(\frac{\theta}{x}\right) \frac{d \theta}{\theta}\right| d x=\int_{1}^{\infty}\left|\int_{n}^{\infty} M(\theta) \rho\left(\frac{x}{\theta}\right) \frac{d \theta}{\theta}\right| \frac{d x}{x^{2}} .
$$

Now we split the outer integral on the right-hand side as $\int_{1}^{n}+\int_{n}^{\infty}$. The first one easily evaluates to $|\gamma(n)| \log n$ taking into account Lemma 2.7(ii) and (iii). This term converges to zero in view of (1.11) and an elementary error term in the prime number theorem. The second one is bounded by

$$
\begin{aligned}
\int_{n}^{\infty} \int_{n}^{\infty}|M(\theta)| \rho\left(\frac{x}{\theta}\right) \frac{d \theta}{\theta} \frac{d x}{x^{2}} & =\int_{n}^{\infty} \frac{|M(\theta)|}{\theta}\left(\int_{n}^{\infty} \rho\left(\frac{x}{\theta}\right) \frac{d x}{x^{2}}\right) d \theta \\
& \ll \int_{n}^{\infty}|M(\theta)| \frac{\log \theta}{\theta^{2}} d \theta \\
& \ll \int_{n}^{\infty} \frac{d \theta}{\theta \log ^{2} \theta} \longrightarrow 0, \quad(n \rightarrow \infty),
\end{aligned}
$$

where we have applied in succession Fubini's theorem, Lemma 3.11, and an elementary error term for the prime number theorem of the form $M(x) \ll x(\log x)^{-3}$.

4. On divergence of certain natural approximations. Throughout this section $1<$ $p<\infty$. All natural approximations considered converge both a.e. and in $L_{1}$ either to $\lambda$ or to $-\chi$, hence not converging in $L_{p}$ to the corresponding generator is equivalent to diverging in $L_{p}$.

4.1. Divergence of approximations to $\lambda$. The main result needs no proof as it is just the counterpositive of statement (d) in Theorem 1.8, namely:

Proposition 4.1. If $\zeta(s)$ has a zero with real part $\geq 1 / p$, then $G_{n}$ diverges in $L_{p}$. In particular $G_{n}$ diverges in $L_{2}$.

REMARK 4.2. This proposition shows that in general the weak implication, (c) $\zeta(s) \neq 0, \mathfrak{R} s>1 / p$ implies $\left\|G_{n}-\lambda\right\|_{r} \rightarrow 0$ for all $r \in(1, p)$ in Theorem 1.8 cannot be made stronger to include $r=p$. The hypothesis of (c) can hold only if $1 \leq p \leq 2$. Although we resolved at the outset to keep $1<p<\infty$, our resolve is weak, so we note that for $p=1$ the strong version is true because of Proposition 3.12. For $p=2$ the strong statement is definitely false for there are zeroes on $\Re s=1 / 2$. In the case $1<p<2$ a simple logical analysis shows that the only interesting case is $\beta=1 / p$. Now, either there are roots on the line $\mathfrak{R}=\beta$, then the strong statement is false; or else, there are no roots on that line, then we can say nothing at present. This is related to Question 2.12.

REMARK 4.3. By Corollary 2.5 there is a subsequence of zero crossings of $\gamma(n)$ where clearly $|\gamma(n)|<1 / n$. For this subsequence the contradiction (3.18) would not hold. Thus the possibility remains open that there is a subsequence of $G_{n}$ that does converge. This peculiarity is common to all natural approximations discussed here. But there are reasons to believe this is may be a mirage. 
To probe a little into the possible mirage we now bring to bear the existence of an isometry (it is actually a unitary operator, but that is not relevant here) of $L_{2}(0, \infty)$ denoted by $U$ in [3] satisfying the following conditions, where we let $\rho_{1}(x)=\rho(1 / x)$ :

$$
\begin{gathered}
U K_{a}=K_{a} U, \quad(a>0), \\
U \rho_{1}(x)=\frac{\rho(x)}{x}, \\
U X(x)=\frac{\sin (2 \pi x)}{\pi x} .
\end{gathered}
$$

For $f \in \mathscr{A}$ as in (1.3),

$$
U f(x)=\frac{1}{x} \sum_{k=1}^{n} c_{k} \theta_{k} \rho\left(\frac{x}{\theta_{k}}\right) .
$$

If we apply this to the Riemann sums of $T f$, when $f$ is continuous of compact support, and make the obvious modifications to the reasoning in Lemma 3.1 and Proposition 3.2, we obtain the following lemma.

LEMMA 4.4. For $f \in L_{2}(0, \infty)$,

$$
U T f(x)=\frac{1}{x} \int_{0}^{\infty} f(\theta) \rho\left(\frac{x}{\theta}\right) d \theta .
$$

Moreover, the right-hand side defines a continuous extension to all $L_{p}(0, \infty)$.

REMARK 4.5. At present we will use this lemma only in $L_{2}$. It is however interesting to see how $U T$ extends to all $L_{p}$ 's given the fact that $U$ cannot be extended continuously to any $L_{p}$ other than for $p=2$ (see [5]). When restricted to $f \in L_{2}(0,1)$ the integral of the right-hand side of (4.5) is the Hilbert-Schmidt operator studied by Alcántara-Bode in [1] where it is shown at the outset that the Riemann Hypothesis is equivalent to the injectivity of this operator.

Lemma 4.4 leads to the simple calculation

$$
U G_{n}(x)=H_{2}(n), \quad \text { for } x \in\left(0, \frac{1}{n}\right),
$$

which spells further trouble for the $L_{2}$ convergence of subsequences of $G_{n}$.

Proposition 4.6. The divergence of $G_{n}$ in $L_{2}$ can be sharpened as follows:

$$
\left\|\lambda-G_{n}\right\|_{2} \gg \max \left(n^{1 / 2} \gamma(n), n^{1 / 2} H_{2}(n)\right) .
$$

REMARK 4.7. Since there are roots of $\zeta(s)$ on $\mathfrak{R} s=1 / 2$, neither $n^{1 / 2} \gamma(n)$ nor $n^{1 / 2} H_{2}(n)$ converge to zero by Corollary 2.5 , and most likely they are unbounded as $n \rightarrow \infty$. However, optimism about almost periodicity of these functions may induce the idea that their zero crossings implied also by Corollary 2.5 will be close together an infinite number of times. 
Proof OF Proposition 4.6. That $\left\|\lambda-G_{n}\right\|_{2} \gg n^{1 / 2} \gamma(n)$ is simply (3.17) for $p=2$. For the second part we use (4.6),

$$
\begin{aligned}
\left\|G_{n}-\lambda\right\|_{2}^{2} & =\left\|U G_{n}-U \lambda\right\|_{2}^{2} \\
& \geq \int_{0}^{1 / n}\left|U G_{n}(x)-U \lambda(x)\right|^{2} d x \\
& \geq \int_{0}^{1 / n}\left|H_{2}(n)-U \lambda(x)\right|^{2} d x \\
& \gg n^{-1}\left|H_{2}(n)\right| .
\end{aligned}
$$

A finer analysis of selected intervals in $(1 / n, \infty)$ seems likely to produce an infinite number of barriers increasing the lower bound in (4.8), so that one may be inclined to think that all subsequences of $G_{n}$ diverge in $L_{2}$.

An even more natural looking approximation of $\lambda$ is obtained by writing the simplest Riemann sum of the integral (3.14), namely

$$
R_{n}(x):=\sum_{k=1}^{n-1} \frac{1}{k} M\left(\frac{n}{k}\right) \rho\left(\frac{k}{n x}\right),
$$

which happens to be a Beurling function in $\mathscr{C}$ with an uncanny resemblance to the dual approximation $F_{n}$ defined by (1.23). But bear in mind that the integral (3.14) is not a proper Riemann integral, and we have not yet been able to show that $R_{n}$ is a natural approximation, in the sense that it satisfies a weak Beurling theorem such as Theorem 1.8, so we state the following true theorem without proof.

Proposition 4.8. $R_{n}$ diverges in $L_{2}$.

Yet another approximation could be defined by truncation, say

$$
T\left(\min \left(n, \max \left(M_{1},-n\right)\right)\right) .
$$

We will not pursue this matter here either, but it seems to deserve some attention.

4.2. Divergence of approximations to $-\chi$. We may treat $S_{n}$ and $V_{n}$ together, defined in (1.22) and (1.21), since $\left\|S_{n}-V_{n}\right\|_{2} \rightarrow 0$. The following proposition is then the corresponding divergence result for $S_{n}$.

Proposition 4.9. If there is some zero of $\zeta(s)$ with real part $1 / p$ then $S_{n}$ and $V_{n}$ diverge in $L_{p}$. In particular $S_{n}$ and $V_{n}$ diverge in $L_{2}$.

Proof. The hypothesis on the zero of $\zeta(s)$ implies, by Corollary 2.5 , that

$$
g(n) \neq o\left(n^{-1 / q}\right) .
$$

Now assume by contradiction that $S_{n}$ converges in $L_{p}$, so it must converge to $-\chi$. On the other hand, noting that $k x>1$ when $x>1 / m$ and $k>m$, we get

$$
\begin{aligned}
\left\|S_{n}-S_{m}\right\|_{p}^{p} & \geq \int_{1 / m}^{\infty}\left|\sum_{k=m+1}^{n} \mu(k) \rho\left(\frac{1}{k x}\right)\right|^{p} d x \\
& =\frac{1}{p-1} m^{p-1}|g(n)-g(m)|^{p} .
\end{aligned}
$$


Then letting $n \rightarrow \infty$ we obtain

$$
\left\|\chi+S_{m}\right\|_{p}^{p} \geq \frac{1}{p-1} m^{p-1}|g(m)|^{p} .
$$

Since the left-hand side goes to zero when $m \rightarrow \infty$ this contradicts (4.11).

REMARK 4.10. Proposition 4.9 implies that in general the weak implication of Balazard-Saias ((i) implies (vii) in [6], see also [14])

$$
\zeta(s) \neq 0, \mathfrak{R} s>1 / p \text { implies }\left\|S_{n}+\chi\right\|_{r} \longrightarrow 0 \quad \forall r \in(1, p)
$$

cannot be made stronger to include $r=p$. An analysis analogous to that carried out for $G_{n}$ in Remark 4.2 is also possible here. Mutatis mutandis the conclusions are the same. But a cautionary note is in order. We have not been able to treat the $L_{p}$ case for $B_{n}$, other than for $p=1$ or 2 .

REMARK 4.11. Again, the existence of a subsequence of zero crossings of $g(n)$ given by Corollary 2.5 indicates that this subsequence is still a candidate in the running to converge in $L_{p}$-norm to $-\chi$. However as with $G_{n}$ we now prove a stricter failure for $S_{n}$ in the $L_{2}$ case.

Proposition 4.12. There exists a constant $C>0$ such that

$$
\left\|x+S_{n}\right\|_{2} \geq \max \left(\frac{C}{\sqrt{n}}|M(n)+2|,|g(n)| \sqrt{n}\right) .
$$

Proof. For $p=2$, inequality (4.13) translates into

$$
\left\|x+S_{n}\right\|_{2} \geq|g(n)| \sqrt{n} .
$$

On the other hand, if we apply $U$ to $S_{n}$ we get

$$
U S_{n}(x)=M(n), \quad\left(0<x<\frac{1}{n}\right) .
$$

Hence

$$
\left\|x+S_{n}\right\|_{2}^{2} \geq \int_{0}^{1 / n}\left|\frac{1}{\pi x} \sin (2 \pi x)+M(n)\right|^{2} d x
$$

and

$$
\left\|x+S_{n}\right\|_{2} \geq C \frac{1}{\sqrt{n}}|M(n)+2|,
$$

for some positive constant $C$.

Odlyzko and te Riele [18] have conjectured that

$$
\limsup _{n \rightarrow \infty} \frac{|M(n)|}{\sqrt{n}}=\infty,
$$

in which case $S_{n}$ would not even be bounded in $L_{2}$, endangering also the possibility of a strong version of condition (vi) in Balazard-Saias's work [6]. On the other hand, by Corollary 2.5 there is a subsequence where $M(n)=-2$, and we know there is a 
subsequence where $g(n)$ crosses zero, with $g(n) \leq 1 / n$. Nevertheless, as for $G_{n}$, one may suspect that there is no $L_{2}$-convergent subsequence of $S_{n}$.

The initial natural approximation $B_{n}$ is more resilient. We already remarked that it is not equivalent to $S_{n}$, neither it is a series as defined in (4.24). The fact that $B_{n}(x)=-1$ in $(1 / n, 1)$ destroys the possibility of using the same argument of Proposition 4.9. However with the help of the operator $U$ we can dispose of the $L_{2}$-case both for $B_{n}$ and $F_{n}$.

Proposition 4.13. Neither $B_{n}$ nor $F_{n}$ converges in $L_{2}$.

Proof. The $U$ defining properties (4.1) and (4.2), as well as (1.11) give $U B_{n}(x)=$ $-n \gamma(n)$ in $(0,1 / n)$. Assume by contradiction that $B_{n}$ converges in $L_{2}$, then so does $U B_{n}$, and therefore

$$
0 \leftarrow-\int_{0}^{1 / n}\left|U B_{n}(x)\right|^{2} d x=n|\gamma(n)|^{2},
$$

which contradicts Corollary 2.5. Likewise the wholly analogous computation $U F_{n}(x)=$ $M(n)-1$ in $(0,1 / n)$ yields the divergence of $F_{n}$ in $L_{2}$.

Analogous considerations as for $S_{n}$ apply in relation to the possibility of a subsequence of $B_{n}$ or of $F_{n}$ converging in $L_{2}$.

To round off the presumption of divergence of the natural approximations in $L_{2}$, we prove Proposition 4.14, a result, suggested by M. Balazard (personal communication), stating that no series of a certain kind in $\mathscr{C}$ can converge to $-\chi$ in $L_{2}$.

Denote by $\mathscr{C}^{\text {nat }}$ the subspace generated by the linearly independent functions $\left\{e_{k} \mid\right.$ $k \geq 2\}$, where

$$
e_{k}(x):=\rho\left(\frac{1}{k x}\right)-\frac{1}{k} \rho\left(\frac{1}{x}\right)
$$

Note that

$$
V_{n}=\sum_{k=2}^{n} \mu(k) e_{k} .
$$

A series in $\mathscr{C}^{\text {nat }}$ is defined as any sequence of type

$$
f_{n}=\sum_{k=2}^{n} c_{k} e_{k}, \quad(n \geq 2) .
$$

We can now state the following proposition.

Proposition 4.14. No series in $G^{\text {nat }}$ converges in $L_{p}(0,1)$ to $-\chi$ if there is a zero of $\zeta(s)$ with real part $1 / p$. In particular, no series in $\mathscr{C}^{\text {nat }}$ converges to $-\chi$ in $L_{2}(0,1)$.

To achieve the proof of this theorem we need a lemma.

LEMMA 4.15. Let $f_{n}$ be a sequence in $\breve{c}^{\text {nat }}$ converging pointwise to $-\chi$. Assume $f_{n}$ is written as

$$
f_{n}(x)=\sum_{k=1}^{n} a_{n, k} \rho\left(\frac{1}{k x}\right),
$$


then

$$
a_{n, j} \longrightarrow \mu(j), \quad(n \rightarrow \infty),
$$

for every $j \geq 1$.

Proof. Each $f_{n} \in \mathscr{C}$, so condition (1.4) implies that it is the right-continuous, step function

$$
f_{n}(x)=-\sum_{k=1}^{n} a_{n, k}\left[\frac{1}{k x}\right],
$$

which is constant on every interval

$$
\left(\frac{1}{j+1}, \frac{1}{j}\right], \quad j=1,2, \ldots
$$

Therefore, pointwise convergence trivially implies

$$
-\lim _{n \rightarrow \infty} f_{n}\left(\frac{1}{j}\right)=\lim _{n \rightarrow \infty} \sum_{k=1}^{n} a_{n, k}\left[\frac{j}{k}\right] \longrightarrow 1, \quad j=1,2, \ldots .
$$

Now we proceed by induction. For $j=1$ it is clear that (4.29) gives $a_{n, 1} \rightarrow 1=\mu(1)$. Next assume for $j>1$ that $a_{n, k} \rightarrow \mu(k)$ for $1 \leq k \leq j-1$, then the limit in (4.29) yields

$$
\sum_{k=1}^{j-1} \mu(j)\left[\frac{j}{k}\right]+a_{n, j} \longrightarrow 1
$$

But comparing this to the well-known

$$
\sum_{k=1}^{j} \mu(j)\left[\frac{j}{k}\right]=1
$$

we obtain the desired $a_{n, j} \rightarrow \mu(j)$ as $n \rightarrow \infty$.

REMARK 4.16. In some sense Lemma 4.15 shows the inevitability of the natural approximation $S_{n}$.

Proof OF Proposition 4.14. We have trivially

$$
f_{n}(x)=-\left(\sum_{k=2}^{n} \frac{c_{k}}{k}\right) \rho\left(\frac{1}{x}\right)+\sum_{k=2}^{n} c_{k} \rho\left(\frac{1}{k x}\right) .
$$

Assume by contradiction that $\left\|\chi+f_{n}\right\|_{p} \rightarrow 0$. For the step functions involved, this clearly implies pointwise convergence, then, from Lemma 4.15 , we get $c_{k}=\mu(k)$ for each $k \geq 2$, which, by the way, forces (4.26) to hold for $k=1$ too. But this immediately implies that $f_{n}=V_{n}$. However, $V_{n}$ diverges in $L_{p}$ by Proposition 4.9 , so we have obtained a contradiction.

ACKNOWLEDGments. The author thanks M. Balazard and E. Saias for useful conversations, and A. M. Odlyzko for his generous help in the matters treated in Section 2. 


\section{REFERENCES}

[1] J. Alcántara-Bode, An integral formulation of the Riemann hypothesis, Mathematisches Institut der Universität Heidelberg, preprint, 1991.

[2] L. Báez-Duarte, On Beurling's real variable reformulation of the Riemann hypothesis, Adv. Math. 101 (1993), no. 1, 10-30.

[3] _ A class of invariant unitary operators, Adv. Math. 144 (1999), no. 1, 1-12.

[4]___ A Möbius convolution and some elementary equivalences with the prime number theorem, preprint, 2000.

[5] - On the type set of the unitary operator U, preprint, 2000.

[6] M. Balazard and E. Saias, Notes sur la fonction $\zeta$ de Riemann. 1 [Notes on the Riemann $\zeta$-function. 1], Adv. Math. 139 (1998), no. 2, 310-321 (French).

[7] _ The Nyman-Beurling equivalent form for the Riemann hypothesis, Exposition. Math. 18 (2000), no. 2, 131-138.

[8] H. Bercovici and C. Foias, A real variable restatement of Riemann's hypothesis, Israel J. Math. 48 (1984), no. 1, 57-68.

[9] A. Beurling, A closure problem related to the Riemann zeta-function, Proc. Nat. Acad. Sci. U.S.A. 41 (1955), 312-314.

[10] A. Blanchard, Initiation à la théorie analytique des nombres premiers, Travaux et Recherches Mathématiques, vol. 19, Dunod, Paris, 1969 (French).

[11] W. F. Donoghue, Distributions and Fourier Transforms, Pure and Applied Mathematics, vol. 32, Academic Press, New York, 1969.

[12] G. H. Hardy, J. E. Littlewood, and G. Pólya, Inequalities, Cambridge Mathematical Library, Cambridge University Press, Cambridge, 1988.

[13] S. V. Konyagin and A. Yu. Popov, On the rate of divergence of some integrals, Mat. Zametki 58 (1995), no. 2, 243-255, translation in Math. Notes 58 (1995), no. 1-2, 841-849 (1996).

[14] J. Lee, Convergence and the Riemann hypothesis, Commun. Korean Math. Soc. 11 (1996), no. 1, 57-62.

[15] N. Levinson, On closure problems and the zeros of the Riemann zeta function, Proc. Amer. Math. Soc. 7 (1956), 838-845.

[16] N. Nikolski, Distance formulae and invariant subspaces, with an application to localization of zeros of the Riemann $\zeta$-function, Ann. Inst. Fourier (Grenoble) 45 (1995), no. 1, 143-159.

[17] B. Nyman, On some groups and semigroups of translations, Thesis, Uppsala University Sweden, 1950.

[18] A. M. Odlyzko and H. J. J. te Riele, Disproof of the Mertens conjecture, J. reine angew. Math. 357 (1985), 138-160.

[19] W. Rudin, Fourier Analysis on Groups, Interscience Tracts in Pure and Applied Mathematics, vol. 12, Interscience Publishers, New York, 1962.

[20] E. C. Titchmarsh, The Theory of the Riemann Zeta-Function, Clarendon Press, Oxford, 1951.

[21] V. I. Vasyunin, On a biorthogonal system associated with the Riemann hypothesis, Algebra i Analiz 7 (1995), no. 3, 118-135, translation in St. Petersburg Math. J. 7 (1996), no. 3, 405-419.

[22] D. V. Widder, The Laplace Transform, Princeton Mathematical Series, vol. 6, Princeton University Press, New Jersey, 1941.

LUIS BÁEZ-DuARTE: DePARTAMENTO DE MATEMÁticas, INSTITUTO VENEZOLANO DE INVESTIGACIONES CiENTÍFICAS, APARTADO 21827, CARACAS 1020-A, VENEZUEla

E-mail address: 1baez@ccs.internet.ve 


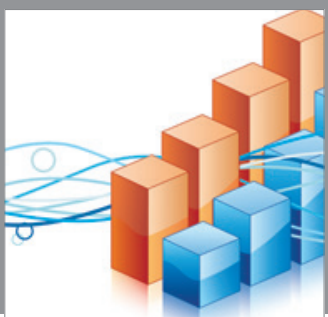

Advances in

Operations Research

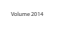

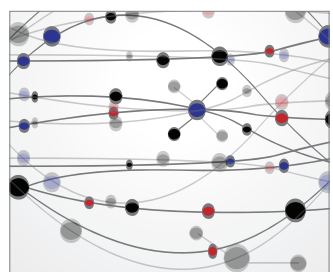

\section{The Scientific} World Journal
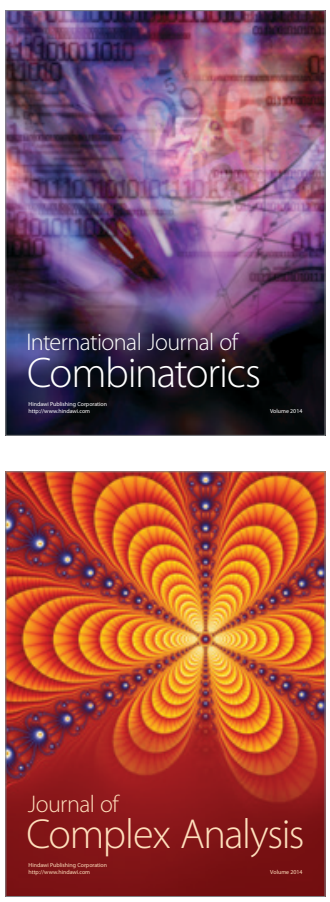

International Journal of

Mathematics and

Mathematical

Sciences
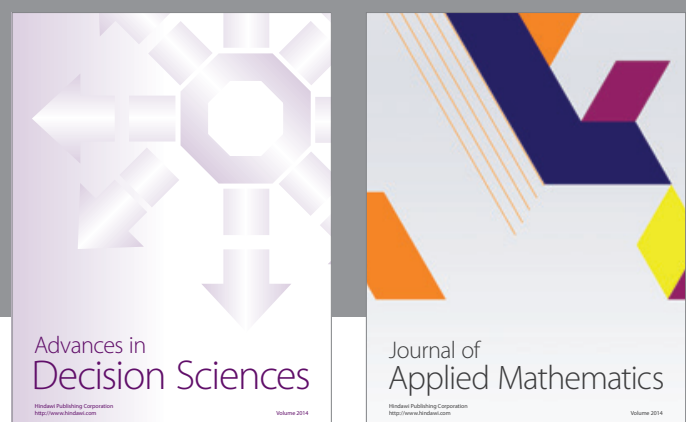

Journal of

Applied Mathematics
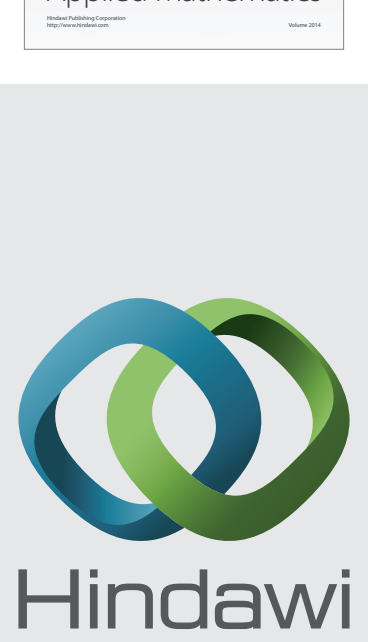

Submit your manuscripts at http://www.hindawi.com
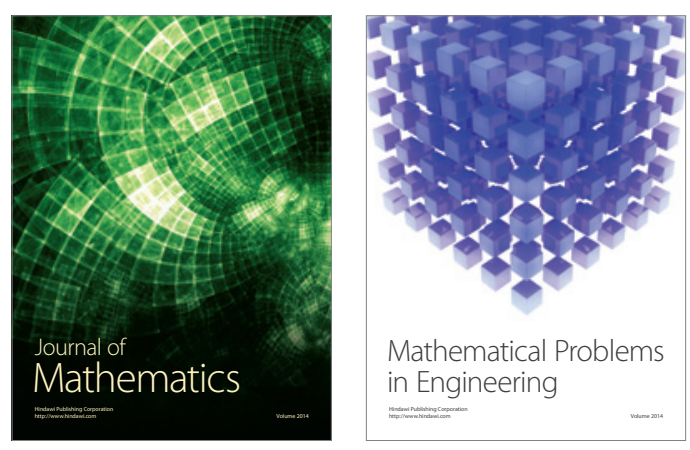

Mathematical Problems in Engineering
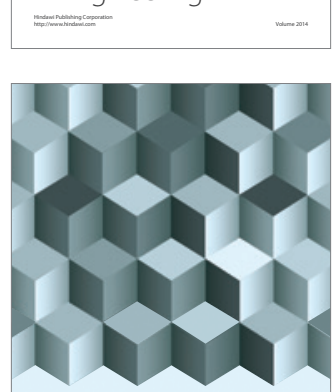

Journal of

Function Spaces
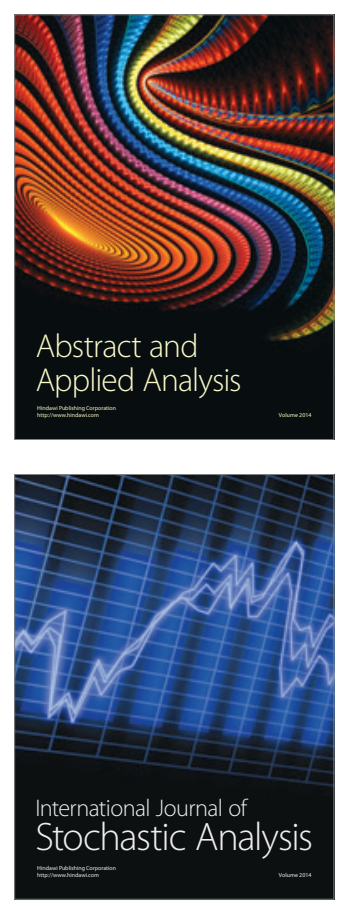

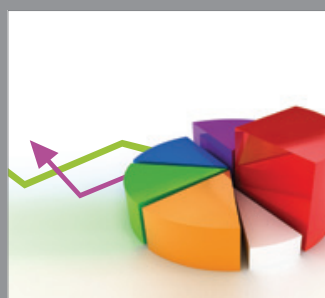

ournal of

Probability and Statistics

Promensencen
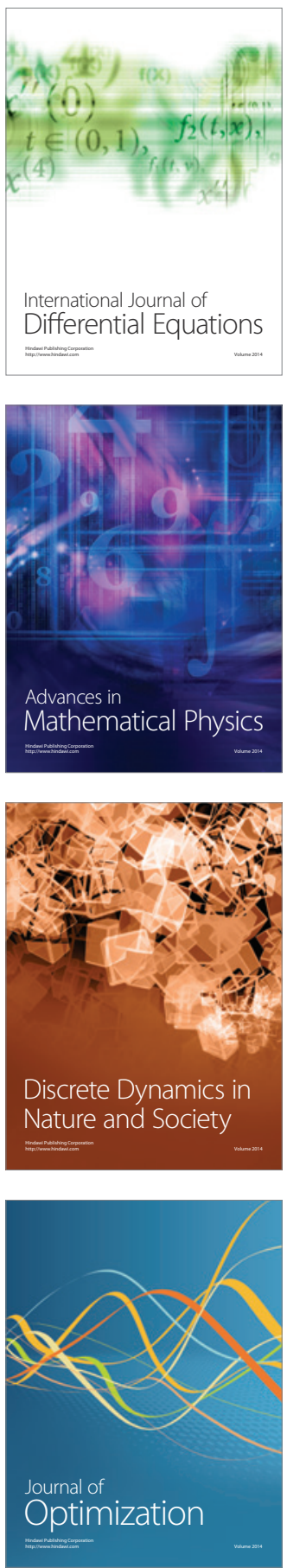